\title{
A NEW PROPOSED APPROACH FOR DENSIFICATION OF THE CONVENTIONAL GEODETIC NETWORKS USING GPS INFORMATION
}

\author{
Rahil A. M., El Gohary A. M., Doma M. I. \\ Civil Engineering Department, Faculty of Engineering, Shebin El-Kom \\ Minoufiya University, Egypt
}

\begin{abstract}
This research paper presents a methodology for densification of conventional geodetic networks by adding GPS points. The feature of this method is to introduce a statistically optimal procedure, based on least squares principle, that allows adjusting the new points, linking it to primary points and connecting it to tie-points with same level neighbor points. The main question to be answered in the current study is how to optimally add stations to the whole geodetic network (existing and added stations)? Basic formulations are given. A numerical example is presented. The obtained results are discussed and analyzed. The standard deviations will be used as a precision criteria.

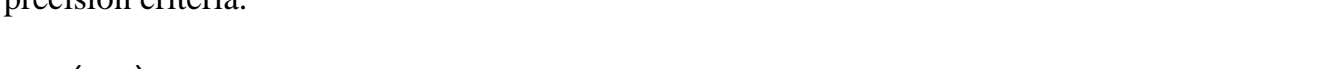

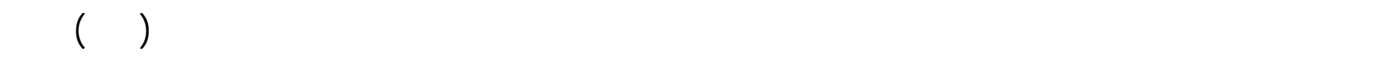

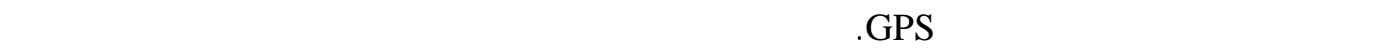

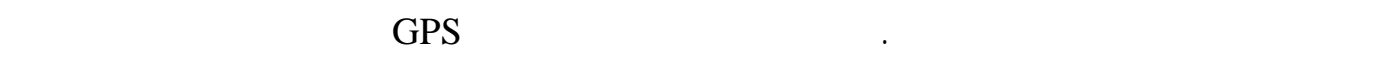

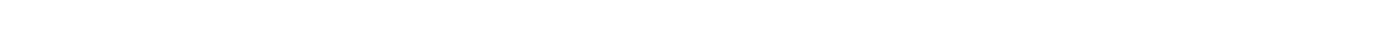
مواضع التط المضافة إلي المبكة الأصلية.
\end{abstract}

\section{INTRODUCTION}

Horizontal terrestrial geodetic control networks are the most important and valuable in each country, because for establishing them time and expenses have been paid. Today the advent of the Global Positioning System (GPS), survey points could, both technically and economically be accessible to use for network densification. Densification is the process by which the number of determined network stations per unit area are increased (e.g. Lugoe, 1990). Dandifying primary project control is one of the major uses of GPS technology. GPS is often more cost-effective, faster, accurate, and reliable than conventional (terrestrial) survey methods. The quality control statistics and large number of redundant measurements of GPS networks help to ensure reliable results. GPS is practically effective for establishing primary control networks as compared with conventional surveys because intervisibility is not required between adjacent stations.

During the past twenty years GPS has been firmly established as a primary tool for the measurement of high-precision geodetic networks. There may be a tendency in some circles to adopt concepts, methods and approaches to geodetic design which were developed basically for classical geodetic measurements. GPS is a precise and reliable tool in a wide range of surveying application. For the establishment and densification of geodetic networks it is the most economical surveying tool (Lavallée, 2006). Practical application of the GPS will depend on (Kebbieh, 1995):

a. Availability of instruments, which looks very favorable in view of decreasing precise and increasing quality of both hardware and software.

b. Availability of precise orbital and other basic data.

c. The willingness of geodesists and surveyors to use this new technique.

\section{MATHEMATICAL MODEL}

The general mathematical model that considers the cases of network densification and integration is one, which relates together the problems of merging multiple sets of existing position information with additional observations. It is the model for the leastsquares adjustment of observations and subsequent computation of positions considering all existing position information in the network.

To put the formulation of the general model in proper perspective, we first regard all existing network stations as having been determined n-times. 
Each determination may have been performed using different positioning systems such as GPS, Doppler, EDM Traversing or Trilateration/Triangulation.

New stations are added during densification to make all existing stations the junction stations of the densification network. A main and auxiliary model will then give the mathematical model. Assume that there is a geodetic network, which has to be observed at two epochs of time $t_{1}$ (conventional observation) and $t_{2}$ (GPS observation), where the second is made to refine the first. There are no constraints as to time intervals between the conventional observation and the GPS observation. For epoch $\left(\mathrm{t}_{1}\right)$, the observation equations model takes the form Schaffrin (2002):

$$
\overline{\mathbf{L}}_{1}=\mathbf{F}_{1}\left(\overline{\mathbf{x}}_{1}, \overline{\mathbf{x}}_{\mathbf{2}}\right)
$$

and for epoch $\left(t_{2}\right)$, the observation equations model takes the form:

where:

$$
\overline{\mathbf{L}}_{2}=\mathbf{F}_{2}\left(\overline{\mathbf{x}}_{2}, \overline{\mathbf{x}}_{3}\right)
$$

$\mathbf{L}_{1}, \mathbf{L}_{2}$ : the adjusted observation vectors at epochs $t_{1}, t_{2}$.

$\mathbf{x}_{\mathbf{1}}=$ Vector of parameters which are considered only at epoch $\mathrm{t}_{1}$, consisting of $\mathrm{u}_{1}$ elements.

$\mathbf{x}_{\mathbf{2}}=$ Vector of parameters of some points of the original plan, which are considered only at epoch $t_{1}$ and $t_{2}$, consisting of $u_{2}$ elements.

$\mathbf{x}_{\mathbf{3}}=$ Vector of parameters of the new points added to the original net and considered only at epoch $t_{2}$, consisting of $\mathrm{u}_{3}$ elements.

The models (1) and (2) take a non-linear form. Remembering that the least squares process deals only with linear models, the above non-linear observation equations must be linearized first which will lead to the following form (e.g., Grafarend, 1974):

$$
\begin{aligned}
& V_{1}=A_{11} x_{1}+A_{12} x_{2}+W_{1} \\
& V_{2}=\quad A_{22} x_{2}+A_{23} x_{3}+W_{2}
\end{aligned}
$$

The above two systems of linearized observation equations should be adjusted simultaneously to allow for correct error propagation for new points $x_{3}$. This could be achieved by using the so-called "dynamic network adjustment” (e.g., Mousa, 1992). This dynamic network adjustment leads not only to correct error propagation for the new points but also to change in the coordinates of the old network points and their covariance matrix as well. This means that the new observations and/or new points influence the old network points which implies that the old network points are considered as free points and hence can get some corrections after adjustment at epoch $\left(t_{2}\right)$

Combining the above linearized systems in a hyper matrix, the following hybrid linearized observation equation can be obtained:

$$
\left(\begin{array}{l}
V_{1} \\
V_{2}
\end{array}\right)=\left(\begin{array}{lll}
A_{11} & A_{12} & 0 \\
0 & A_{22} & A_{23}
\end{array}\right)\left(\begin{array}{l}
x_{1} \\
x_{2} \\
x_{3}
\end{array}\right)+\left(\begin{array}{l}
W_{1} \\
W_{2}
\end{array}\right)
$$

The corresponding hyper weight matrix (P) will be given as:

$$
\mathbf{P}=\left(\begin{array}{cc}
\Sigma_{\mathbf{L}_{1}}^{-1} & 0 \\
0 & \Sigma_{\mathbf{L}_{2}}^{-1}
\end{array}\right)
$$

Applying the least squares principle $\left(\mathrm{V}^{\mathrm{T}} \mathrm{PV}=\right.$ min.) on the hybrid system given by equation (5), the matrix of the normal equation can be obtained and solved, then, the adjustment process is completed to get the estimated $\mathrm{x}_{1}, \mathrm{x}_{2}, \mathrm{x}_{3}$ and their corresponding variance covariance matrices.

By investigating the results from the adjustment process, the estimated parameters and strength of the network can be compared with the required specifications, according to the measures of precision.

\section{GPS BASELINE VECTOR}

Let us consider a small network composed of four control points numbered A, B, C, D and a single secondary (new) point $\mathrm{F}$. The coordinates of point $\mathrm{F}$ can be determined on the basis of four independent vectors measured by GPS.

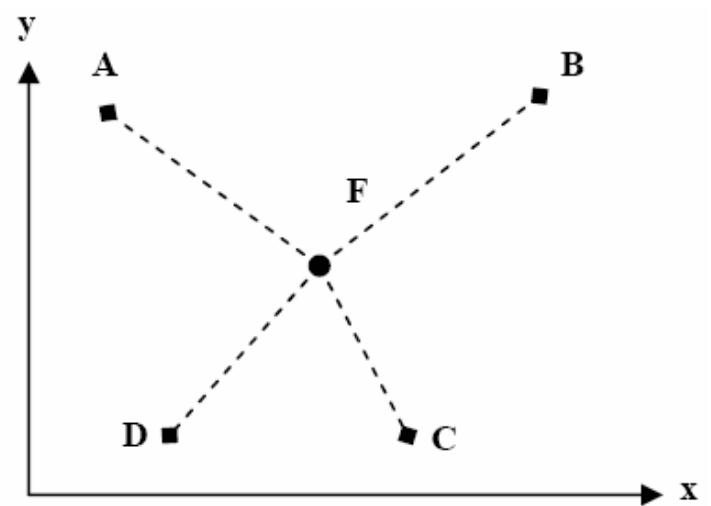

Figure (1): Approximate geometry of the GPS network

A measured GPS vector is the difference in geocentric coordinates of its end points. In most cases, we transform the vector from geocentric (WGS84) to a local geodetic horizontal system.

The mathematical model of GPS measurements is linear. The elements of the design matrix (A), in terms of geocentric coordinates as well as in terms of the respective local horizon coordinates, are invariably: 0,1 or -1 . We can notice a resemblance between the design matrix of a GPS network and that of a leveling network. In both cases the design 
matrices are independent of network geometry (Even-Tzur, 1996; Stopar, 2001).

We continue now with the above network, where the first four points will be treated as control points with fixed (known) coordinates. Estimation of the coordinates of point $F$ will be based on four GPS vectors measured between point $F$ and points $A, B, C$ and D.

The transposed design matrix A will be:

$$
\mathbf{A}^{\mathbf{T}}=\left[\begin{array}{llllllllllll}
\mathbf{1} & \mathbf{0} & \mathbf{0} & \mathbf{1} & \mathbf{0} & \mathbf{0} & \mathbf{1} & \mathbf{0} & \mathbf{0} & \mathbf{1} & \mathbf{0} & \mathbf{0} \\
\mathbf{0} & \mathbf{1} & \mathbf{0} & \mathbf{0} & \mathbf{1} & \mathbf{0} & \mathbf{0} & \mathbf{1} & \mathbf{0} & \mathbf{0} & \mathbf{1} & \mathbf{0} \\
\mathbf{0} & \mathbf{0} & \mathbf{1} & \mathbf{0} & \mathbf{0} & \mathbf{1} & \mathbf{0} & \mathbf{0} & \mathbf{1} & \mathbf{0} & \mathbf{0} & \mathbf{1}
\end{array}\right]
$$

\section{GEODETIC CONTROL AND DATUM DEFINITION}

In order to understand GPS and its positional information, it is important to understand the reference system on which it is based, and how that reference system relates to the user's local system. The GPS satellites are referenced to the World Geodetic System of 1984 (WGS 84) ellipsoid (US Army Corps, 2003; Kumar, 2006). For surveying purposes, this earth-centered WGS 84 coordinate system must be converted (i.e., transformed) to a user defined ellipsoid and datum, such as the North American Datum of 1983 (NAD 83), the North American Vertical Datum of 1988 (NAVD 88), or National Geodetic Vertical Datum of 1929 (NGVD 29). Differential positioning partially provides this transformation by locating one of the receivers at a known point on the user's reference datum or frame. However, for more precise applications, the reference datum cannot be considered as absolutely rigid in time (US Army Corps, 2003).

Observation equations relating to any geodetic network are normally rank-deficient due to the lack of datum reference. There is, therefore, a need to define the datum in order to solve for the positioning parameters. Datum definition can be accomplished by a careful selection of some constraints added to the observation equation model. This could be done while fixing one or more reference stations during the adjustment procedure. When only one reference station is held fixed we establish a datum referred to as a minimum constraint solution; if more than one station is fixed, the datum will be referred to as an over-constrained solution (Kashani, 2003). For a free network approach, it is often desirable that the property of the minimum sum of the variances and minimum sum of squares of the correction to the coordinates is restricted to certain points (Kock, 1999).

In the best fitting process the high accuracy GPS network might be deteriorated by the existing local terrestrial network, the latter usually being of lower accuracy.

A sufficient network transformation to the local datum applied in practice can be obtained by the following algorithm (Fotiou and Livieratos 2000, Rossikopoulos 1999):

A. Determination of 2-D transformation parameters by means of common points:

1. Transform the Cartesian GPS (x, y, z) coordinates to ( $\mathrm{x}, \mathrm{y}, \mathrm{z}$ )LD of local datum by means of approximate shift parameters and/or other known parameters.

2. From (x, y, z) $)_{\mathrm{LD}}$ compute ellipsoidal $(\Phi, \lambda$, $\mathrm{h})_{\mathrm{LD}}$ coordinates.

3. From $(\Phi, \lambda)_{\mathrm{LD}}$ compute $(\mathrm{E}, \mathrm{N})_{\mathrm{LD}}$ plane coordinates by means of mapping equations.

4. Determine the 2-D transformation parameters between the approximately

B. Transformation of non-common points:

1. Transformation the non-common GPS Cartesian coordinates using the above 1, 2, 3 steps and used model with estimated transformation parameters from step A4.

In case of an acceptable transformation model for the area under consideration exists, the corresponding model is applied. A 3-D transformation model might also be directly applied between GPS and local Cartesian coordinates, skipping the previous A1 to A4 steps. In this case, the geoidal heights in the local datum should be characterized by such an accuracy like that of the local plane coordinates the latter being usually of higher accuracy.

In this paper a numerical example is presented consisting of a 2-D over-constrained network adjustment, where a rigorous incorporation to local datum is achieved without using a transformation model.

\section{THE STEPS OF SOLUTION FOR THE DESIFICATION}

The solution steps for the proposed approach will be given below

Given:

1- The positions of the existing points (coordinate components),

2- The observational plan for the existing points (trilateration, triangulation or hybrid network),

3- The case of network (free, minimum constraints or overconstrained),

3- The positions of the added points (GPS).

4- The observing plan of the added points.

\section{Required:}

Final criteria of the final geodetic network (conventional instruments and GPS). 
- The solution steps of the case-study can be summarized as follows:

a) Computation of the design matrix $\left(\mathrm{A}_{1}\right)$ for the conventional instruments

This can be achieved, by using the given coordinates of the existing points, to evaluate the partial derivatives within the matrix $\left(\mathrm{A}_{1}\right)$.

b) Computation of the design matrix $\left(\mathrm{A}_{2}\right)$ for the GPS observation

This will be achieved, by using the given coordinates of the added points to evaluate the partial derivatives within the matrix $\left(\mathrm{A}_{2}\right)$,

c) Computation of the design matrix (A) for the combination of the observations

The combination design matrix can be written as:

$$
\mathbf{A}=\left(\begin{array}{l}
\mathbf{A}_{1} \\
\mathbf{A}_{2}
\end{array}\right)
$$

d) Computation of an actual weight matrix $\left(\mathrm{P}_{\underline{a}}\right)$ for the existing stations:

The weight matrix $\left(\mathrm{P}_{\mathrm{a}}\right)$ of conventional geodetic network can be computed from an observational plan for existing stations.

e) Computation of a weight matrix $\left(\mathrm{P}_{\mathrm{GPS}}\right)$ for the existing stations:

In the current study, we assume that accuracies of the horizontal components of the measurements vectors $(\mathrm{x}, \mathrm{y})$ and the accuracy of the vertical component $(\mathrm{z})$ are equal.

f) Computation of a weight matrix (P) for the combination of the observations:

The final weight matrix for both conventional observations and GPS survey can be expressed by:

$$
\mathbf{P}=\left(\begin{array}{cc}
\mathbf{P}_{\mathrm{a}} & \mathbf{0} \\
\mathbf{0} & \mathbf{P}_{\text {GPS }}
\end{array}\right)
$$

g) Calculation of the final variance-covariance matrix $\left(\mathrm{C}_{\mathrm{f}}\right)$ :

The variance-covariance matrix of the estimated coordinates can be classified into three main types (according to the type of network under study):

1) Minimum constraints networks

$$
\mathbf{C}_{\mathbf{f}}=\left(\mathbf{A}^{\mathrm{T}} \mathbf{P} \mathbf{A}+\mathbf{D D}^{\mathrm{T}}\right)^{-1}-\mathbf{H}\left(\mathbf{H}^{\mathrm{T}} \mathbf{D} \mathbf{D}^{\mathrm{T}} \mathbf{H}\right)^{-1} \mathbf{H}^{\mathrm{T}}
$$

2) Free network concept: the datum matrix (D) $=0$, and hence, the variance-covariance matrix can be computed by:

$$
\mathbf{C}_{\mathbf{f}}=\left(\mathbf{A}^{\mathbf{T}} \mathbf{P} \mathbf{A}\right)^{+}
$$

3) Overconstrained networks: the variancecovariance matrix can be computed by:

$$
\mathbf{C}_{\mathbf{f}}=\sigma_{0}^{2}\left(\tilde{\mathbf{A}}^{\mathbf{T}} \mathbf{P} \tilde{\mathbf{A}}\right)^{-1}
$$

Using the Pc-Matlab version with a personal computer, the program has been devoted to determine the densification of the conventional geodetic network by adding GPS information; this Matlab program was designed, tested and written by the authors.

\section{APPLICATION OF THE PROPOSED ALGORITHM}

Let us assume that we have measured a conventional trilateration geodetic network with a layout as shown in Figure (2) and use only EDM instrument with achievable accuracy $\sigma_{\mathrm{s}}^{2}=(\mathbf{0 . 2} \mathbf{p p m} . \mathbf{S})^{2}$, where $S$ is the distance computed from the approximate coordinates. After a trilateration observed, one wants to densify this network, the 7-points GPS will be added to this network with a layout as shown in Figure (3). Ninety-one independent GPS vectors can be measured. Suppose that the selected GPS receivers can allow for a baseline to be determined with the following precision:

$$
\sigma_{S}^{2}=(2)^{2} \mathrm{~mm}^{2}+(1 \mathrm{ppm} . \mathrm{S})^{2}
$$

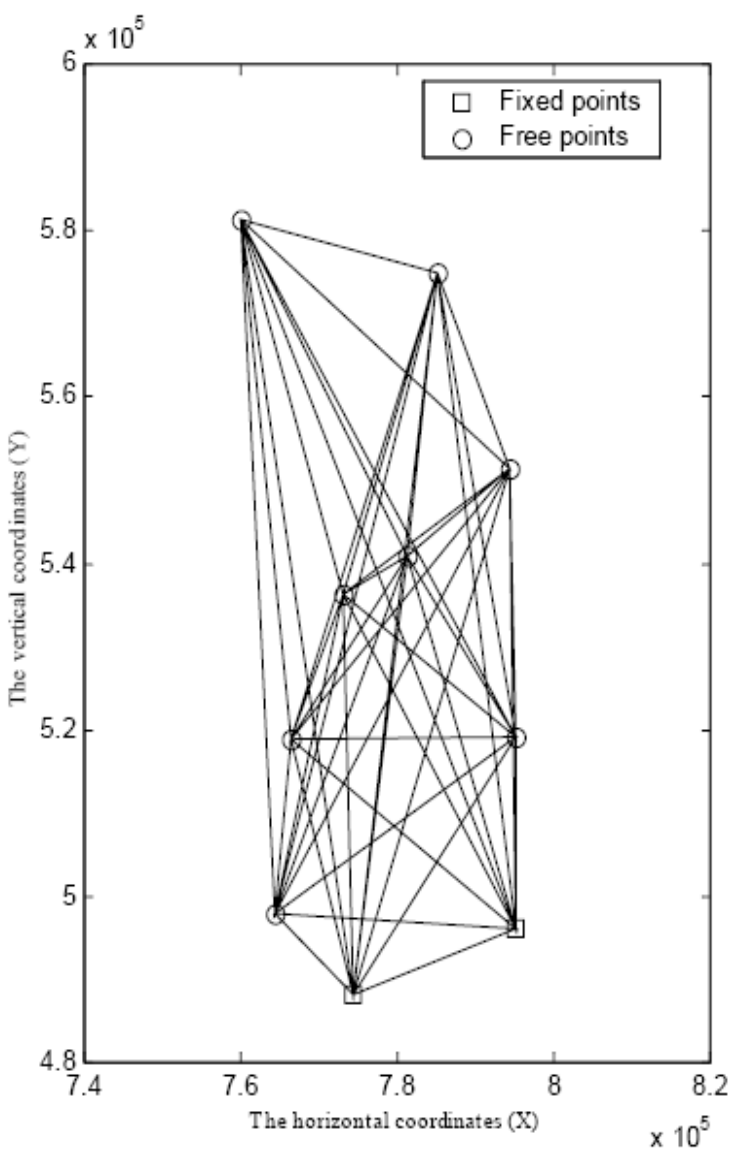

Figure (2): Existing stations of a trilateration network 


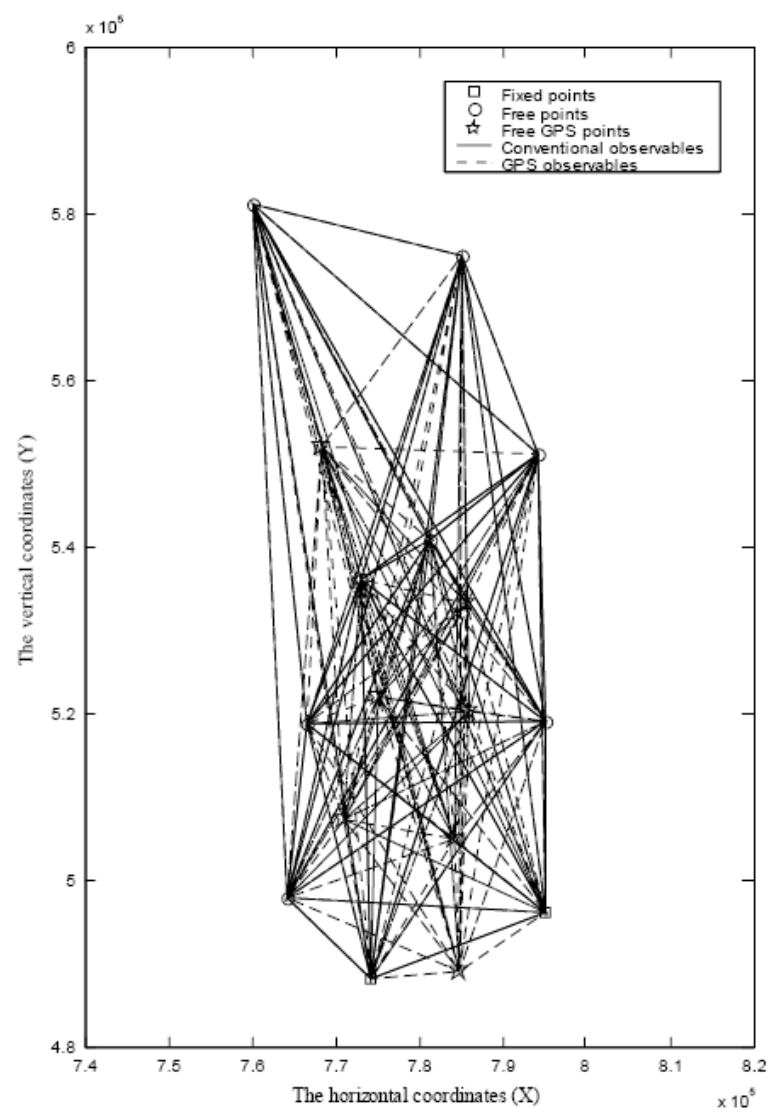

Figure (3): The existing and added (GPS) netpoints

\section{DISCUSSION OF THE OBTAINED RESULTS:}

The results of this case study will be obtained from the over-constrained networks concept. Also, the precision criteria, formed as a criterion matrix, will be considered.

Tables (1) and (2) include the precision before and after densification. The results showed that:

a) The standard deviations of the existing points before applying the densification are ranged from $3.60 \mathrm{~mm}$ at point $\mathrm{P}_{4}$ to $21.98 \mathrm{~mm}$ at point $\mathrm{P}_{9}$, while the standard deviations of the existing points after the densification are ranged from $4.92 \mathrm{~mm}$ at point $\mathrm{P}_{5}$ to $14.46 \mathrm{~mm}$ at point $\mathrm{P}_{9}$.

b) The ratio between the standard deviation in $\mathrm{x}$-axis $\left(\boldsymbol{\sigma}_{\mathbf{x}}\right)$ and the standard deviation in $\mathrm{y}$ - axis $\left(\boldsymbol{\sigma}_{\mathbf{y}}\right)$ before applying the densification procedure are ranged between 0.92 at point $\mathrm{P}_{3}$ to 4.89 at point $\mathrm{P}_{10}$, while The ratio between the standard deviation in $\mathrm{x}$-axis $\left(\boldsymbol{\sigma}_{\mathbf{x}}\right)$ and the standard deviation in $\mathrm{y}$ - axis $\left(\boldsymbol{\sigma}_{\mathrm{y}}\right)$ after applying the densification procedure are ranged between 1.00 at point $\mathrm{D}_{1}$ to 1.61 at point $\mathrm{P}_{10}$, that means the homogeny and isotropy of the network become good after the densification apply.
Table (1): The actual coordinate components of network existing points, their standard deviations and the ratio between the standard deviations

\begin{tabular}{|c|c|c|c|c|c|}
\hline \multirow{2}{*}{ Point } & \multicolumn{2}{|c|}{$\begin{array}{l}\text { Actual coordinates } \\
\text { of netpoints }\end{array}$} & \multicolumn{3}{|c|}{ Achievable precision } \\
\hline & $\begin{array}{c}\mathbf{x} \\
(\mathbf{m})\end{array}$ & $\begin{array}{c}\mathbf{y} \\
(\mathbf{m})\end{array}$ & $\begin{array}{c}\sigma \mathbf{X} \\
(\mathbf{m m})\end{array}$ & $\begin{array}{c}\sigma_{\mathbf{y}} \\
(\mathbf{m m})\end{array}$ & $\frac{\sigma_{x}}{\sigma_{y}}$ \\
\hline $\mathrm{P}_{1}$ & 774309 & 488122 & Fixed & Fixed & Fixed \\
\hline $\mathrm{P}_{2}$ & 795112 & 496201 & Fixed & Fixed & Fixed \\
\hline $\mathrm{P}_{3}$ & 764268 & 497939 & 4.31 & 4.71 & 0.92 \\
\hline $\mathrm{P}_{4}$ & 795094 & 519243 & 7.41 & 3.60 & 2.06 \\
\hline $\mathrm{P}_{5}$ & 766535 & 518907 & 7.47 & 4.73 & 1.58 \\
\hline $\mathrm{P}_{6}$ & 773001 & 536222 & 10.04 & 4.29 & 2.34 \\
\hline $\mathrm{P}_{7}$ & 781231 & 541021 & 13.15 & 5.05 & 2.60 \\
\hline $\mathrm{P}_{8}$ & 794212 & 551322 & 10.84 & 3.77 & 2.88 \\
\hline $\mathrm{P}_{9}$ & 760010 & 581222 & 21.98 & 8.90 & 2.47 \\
\hline $\mathrm{P}_{10}$ & 785111 & 575022 & 20.18 & 4.31 & 4.89 \\
\hline
\end{tabular}

Table (2): The coordinate components of final points (existing and adding of network points) and their standard deviations

\begin{tabular}{|c|c|c|c|c|c|}
\hline \multirow[t]{2}{*}{ Point } & \multicolumn{2}{|c|}{$\begin{array}{l}\text { Simulated } \\
\text { coordinates of } \\
\text { netpoints }\end{array}$} & \multicolumn{3}{|c|}{ Achievable precision } \\
\hline & $\begin{array}{c}\mathbf{x} \\
(\mathbf{m})\end{array}$ & $\begin{array}{c}\mathbf{y} \\
(\mathbf{m})\end{array}$ & $\begin{array}{c}\sigma_{\mathbf{x}} \\
(\mathbf{m m})\end{array}$ & $\begin{array}{c}\sigma_{\mathrm{y}} \\
(\mathrm{mm})\end{array}$ & $\frac{\sigma_{x}}{\sigma_{y}}$ \\
\hline $\mathrm{P}_{1}$ & 774309 & 488122 & Fixed & Fixed & Fixed \\
\hline $\mathrm{P}_{2}$ & 795112 & 496201 & Fixed & Fixed & Fixed \\
\hline $\mathrm{P}_{3}$ & 764268 & 497939 & 5.94 & 5.51 & 1.08 \\
\hline $\mathrm{P}_{4}$ & 795094 & 519243 & 5.81 & 5.12 & 1.13 \\
\hline $\mathrm{P}_{5}$ & 766535 & 518907 & 5.82 & 4.92 & 1.18 \\
\hline $\mathrm{P}_{6}$ & 773001 & 536222 & 5.89 & 5.43 & 1.09 \\
\hline $\mathrm{P}_{7}$ & 794212 & 551322 & 8.29 & 7.23 & 1.15 \\
\hline $\mathrm{P}_{8}$ & 781231 & 541021 & 5.88 & 5.47 & 1.08 \\
\hline $\mathrm{P}_{9}$ & 760010 & 581222 & 14.46 & 11.76 & 1.23 \\
\hline $\mathrm{P}_{10}$ & 785111 & 575022 & 13.29 & 8.28 & 1.61 \\
\hline $\mathrm{D}_{1}$ & 784998 & 489124 & 6.02 & 6.02 & 1.00 \\
\hline $\mathrm{D}_{2}$ & 771022 & 507231 & 5.48 & 5.47 & 1.00 \\
\hline$D_{3}$ & 784351 & 505121 & 5.48 & 5.48 & 1.00 \\
\hline $\mathrm{D}_{4}$ & 786142 & 520151 & 5.03 & 4.98 & 1.01 \\
\hline $\mathrm{D}_{5}$ & 775123 & 522163 & 5.02 & 4.97 & 1.01 \\
\hline$D_{6}$ & 785755 & 533211 & 5.50 & 5.34 & 1.03 \\
\hline $\mathrm{D}_{7}$ & 768500 & 552151 & 8.16 & 7.92 & 1.03 \\
\hline
\end{tabular}

\section{CONCLUDING REMARKS}

Depending on the obtained results and the analysis carried out on it, we can summarize the following conclusions:

- The present mathematical model for densification of the conventional geodetic networks will be modified the precision of the points (in this current 
numerical example the standard deviations of the points will be used as precision).

- The homogeneity and isotropy for the existing points will be increased after applying the densification procedure.

- GPS is practically effective for adding control networks as compared with conventional surveys because intervisibility is not required between adjacent stations, this gives the user a lot of freedom for choosing the position of added points in the final network scheme.

\section{REFRENCES}

Even-Tzur, G. and Papo, H.B. (1996). "Optimization of GPS Network By Linear Programming” Survey Review, 33, 264,October 1996, pp 537-545.

Fotiou, A. and Livieratos E. (2000). "Geometric Geodesy and Networks” Aristotle University of Thessaloniki, Editions Ziti, Thessaloniki, Greece.

Grafarend, E.W. (1974). "Optimization of geodetic network.” Bolletino di Geodesia a Science Affini, vol. 33,No. 4 ; pp 351 - 406.

Kashani, I., P. Wielgosz and D. Grejner-Brzezinska (2003). "Datum Definition in the Long Range Instantaneous RTK GPS Network Solution" Journal of Global Positioning System (2003) vol. 2, No. 2; 100-108.

Kebbieh, Y.K. (1995). “ The Role of GPS Survey in Geodetic Control Networks” M. Sc. Thesis, Faculty of Engineering, Cairo University, Giza, Egypt.

Koch, K.R.(1999). "Parameter Estimation and Hypothesis Testing in Linear Models"
Spring Verlag, Berlin Heidelberg New York, 333p.

Kumar, M. and Reilly, J.P. (2006). "Is definition of WGS84 Correct” Coordinates, Volume П, Issue 12, December 2006.

Lugeo, F.N. (1990). "Regorious Mathematical Models For the Densification and Integration of Geodetic Networks.” Bulletin Geodesique, No. 64, pp. 219-229.

Lavallée A.D., Dam T.V., Blewitt G., and Clarke P.J. (2006). "Geocenter motions from GPS: A unified observation model” Journal of Geophysical Research. Vol. 111, B05405, doi: 10.1029/2005JB0037 84, 2006.

Mousa, A.K. (1992). "Design of geodetic networks for monitoring recent crustal movements using the optimization theory.” M.Sc. Thesis, Faculty of Engineering, Ain Shams University, Cairo, Egypt.

Rossikopoulos, D. (1999). “surveying Networks and Computations" Aristotle University of Thessaloniki, Editions Ziti, Thessaloniki, Greece.

Schaffrin, B., 2002. "Reproducing estimates via least squares: An optimal alternative to the Helmert transformation” In: E.W. Grafarend, F.W. Krumm, and V.S. Schwarz (eds.). Geodesy - The Challenge of the 3rd Millennium, Springer, Berlin/ Heidelberg/ New York: 387-392.

Stopar, B. (2001). "Second Order Design of Horizontal GPS net” Survey Review, 36, 4453. (January 2001).

US Army Corps of Engineering (2003). "NAVSTAR Global Positioning System Surveying EM 1110-1-1003”, Department of the Army, Washington.(July 2003). 